\title{
Pruebas T vs análisis categorial: evolución de la agilidad en los adultos mayores tras dos años de entrenamiento \\ T test vs categorical analysis: evolution of agility in older adults following two years of training \\ *Ana Cordellat Marzal, *Ainoa Roldán Aliaga, **Pablo Monteagudo Chiner, *Anabel Forte Deltell, *Cristina Blasco- Lafarga \\ *Universidad de Valencia (España), **Universidad Jaume I (España)
}

\begin{abstract}
Resumen. Entendiendo que entrenar no siempre significa alcanzar la independencia funcional para un adulto mayor (AM), ni desentrenar perderla, se crearon 4 categorías para valorar el efecto a largo plazo de un programa multicomponente físico-cognitivo sobre el nivel de independencia en sus usuarios. Se evaluaron 25 AM sanos (69.74 \pm 5.36 años) a lo largo de dos años, considerando 2 periodos de Entrenamiento (E1, E2; 8 meses cada uno) y Desentrenamiento (D1, D2; 3 meses + 1 de evaluación). Se analizó la agilidad (Time Up\&Go Test) por su relación con la marcha y la prevención de caídas. Los cambios de categoría entre periodos se analizaron mediante el test exacto de Fisher, seguido de un ANOVA de medidas repetidas para comparar el análisis tras ambos enfoques. Aunque la agilidad mostró una mejora significativa al entrenar, mayor en E1 que en E2, el porcentaje de participantes independientes funcionalmente fue mayor en E2. En términos de desentrenamiento, la comparación por pares mostró pérdidas superiores durante D2, aunque esto no impidió que más de la mitad de los participantes alcanzaran la independencia funcional en este segundo periodo. Ambos análisis son complementarios y clarifican el beneficio del programa a largo plazo en la agilidad de la población adulta.

Palabras clave: envejecimiento, desentrenamiento, doble tarea, independencia funcional.
\end{abstract}

\begin{abstract}
Abstrat. Exercise training does not ensure functional independence among elders, neither detraining means to lose it, so 4 categories where designed in order to analyse the long-term effects of a multicomponent physical-cognitive training program over their users' functional independence. A total of 25 healthy elders ( $69.74 \pm 5.36$ years old) were assessed during two years, including 2 periods of training (T1, T2; 8 months each one) and detraining (D1, D2; 3 months +1 of testing). Agility (Time Up\&Go Test) was analysed because of its relationship with gait and fall-prevention. The exact Fisher's test was considered to analzse between-period category changes, followed by a Repeated Measures ANOVA, to compare the results in both approaches. Although agility showed significant improvements following training, larger in T1 compared to T2, the percentage of independent participants was higher in T2. In terms of detraining, paired comparison showed larger losses during D2. However, more than half of participants reached the physical independence during this second period. Both analyses are complementary and clarify the long-term benefits of this training program for the agility of the elderly population.
\end{abstract}

Key words: aging, detraining, dual task, functional independence.

\section{Introducción}

Frente a los nuevos retos económicos y sociales que supone el aumento de la población española mayor de 65 años, se requiere la promoción de la actividad física como forma de frenar el deterioro funcional que causa el envejecimiento (Pedersen y Saltin, 2015). Así, multitud de estudios han demostrado que el ejercicio físico es una herramienta no farmacológica capaz de enlentecer dichas pérdidas (Bauman, Merom, Bull, Buchner, y Fiatarone Singh, 2016; Martínez-Heredia, Santaella, y Rodríguez-García, 2021; Salinas-Martínez, Cocca, Mohamed, y Viciana, 2010), además de prevenir la dependencia funcional.

En la literatura se ha propuesto la evaluación de la velocidad de paso como una forma fácil, segura y sencilla para valorar la funcionalidad y el equilibrio en los adultos mayores (Fritz y Lusardi, 2009). Sin embargo, la marcha es una tarea de control complejo que requiere de procesos que incluyen al sistema motor, perceptual y cognitivo (Fritz y Lusardi, 2009), junto a las demandas cardiorrespiratorias, y por ello merece especial atención y cuidado.

En este contexto, la agilidad -también conocida como equilibrio dinámico- gana importancia en los programas de ejercicio para adultos mayores, pues es igualmente compleja,

Fecha recepción: 18-02-20. Fecha de aceptación: 28-04-20

Ana Cordellat Marzal

ana.cordellat@uv.es interacción de otras capacidades como el equilibrio y la fuerza (Donath, van Dieën, y Faude, 2016). La agilidad repercute de forma importante en la autonomía de estos mayores, y particularmente, el test de levantarse, dar la vuelta al cono y sentarse, conocido como «Time Up\&Go» test (TUG), también ofrece información acerca de la marcha y la prevención de caídas (Sampedro, Meléndez, y Ruiz, 2010). Además, la literatura establece que realizar dicha prueba por encima de 10 segundos se relaciona con un mayor número de comorbilidades, con el consiguiente riesgo para la salud (Lee et al., 2020). Por tanto, el TUG se propone como una prueba sencilla y rápida de ejecutar que aporta información muy valiosa. Y de la misma forma nos permite categorizar a los mayores de nuestros programas de entrenamiento, ya que existen numerosos trabajos con valores de referencia (Rikli y Jones, 2013) que señalan cuáles son los valores óptimos para género y edad, aportando una mayor información a los técnicos/entrenadores.

Por otro lado, en cuanto a los programas de entrenamiento, existen multitud de intervenciones, pero debido a la complejidad e interacción entre sistemas, aquellos basados en el trabajo de la marcha, equilibrio y fuerza, conocidos como entrenamiento multicomponente, ayudan a la mejora holística del individuo (Bouaziz et al., 2016), con mejoras sobre las capacidades condicionales y complejas, así como en la percepción de la calidad de vida. Y sobre ellos han surgido, recientemente, los que añaden tareas cognitivas como pueden ser el baile o con videojuegos (Eggenberger, 
Theill, Holenstein, Schumacher, y de Bruin, 2015; Gerard, Salicetti, Moncada, y Solano, 2018), que además de mejoras en la función física también las obtienen sobre la función cognitiva. Pero no debe perderse de vista que el fin último del ejercicio en esta población es mantenerse independiente el mayor tiempo posible, por lo que será tan necesario conocer los beneficios del entrenamiento sobre la autonomía de los participantes, como la repercusión del desentrenamiento o la forma en que se van perdiendo estos beneficios durante el cese de la actividad, con posibles repercusiones sobre la citada independencia. De hecho el concepto de «Aging in Place» recuerda la importancia de poder envejecer en el hogar, de forma segura e independiente (Wiles, Leibing, Guberman, Reeve, y Allen, 2012).

Así pues, el presente trabajo tiene como objetivo observar los efectos del entrenamiento y el desentrenamiento sobre la agilidad, en un grupo de adultos mayores sanos que participaron en el programa EFAM-UVC a lo largo de dos años. Se analiza para ello los cambios tras dos periodos de entrenamiento y sus desentrenamientos. Igualmente, se realiza un análisis categorial para analizar el impacto y evolución del programa en términos de cercanía o alejamiento a los estándares de independencia funcional propuestos por Rikli y Jones (2013), considerando cada género y grupo de edad. Como hipótesis de partida se considera: 1) que el programa EFAM-UVC conseguirá mejorar la agilidad en sus dos periodos de entrenamiento, con pérdidas mayores tras el primer desentrenamiento; 2) que los cambios en cada periodo repercutirán sobre los niveles de independencia de los participantes; y, sobre todo, 3) que ambos enfoques se complementan y mejoran el conocimiento sobre la evolución de la agilidad en la población adulta.

\section{Material y método}

\section{Protocolo y población de estudio}

Ochenta y nueve adultos mayores sanos, usuarios del programa EFAM-UVC iniciaron este estudio, con seguimiento longitudinal de dos años. De los 49 que fueron regulares y completaron el entrenamiento y su evaluación en el primer curso, 47 reanudaron el programa, pero sólo 25 realizaron todos los test, tanto al final de segundo como al inicio del que sería su tercer curso, constituyendo la muestra final (edad media 69.74 $\pm 5.36,84 \%$ mujeres). Los criterios de inclusión fueron tener más de 65 años; no padecer contraindicación expresa para la práctica de ejercicio físico; haber asistido al menos a un $70 \%$ de las sesiones; y haber completado dos cursos consecutivos, iniciando un tercero. Todos los participantes, que asistieron voluntariamente al programa debido al contacto con centro de atención especializado en el mayor, recibieron amplia información y firmaron el consentimiento informado, aprobado por el comité de ética de la Universidad de Valencia (H1363126067752).

Más que un programa cerrado, EFAM-UVC) (BlascoLafarga et al., 2016) es una metodología de entrenamiento funcional cognitivo neuromotor, diseñada especialmente para mejorar la condición física en los adultos mayores sujetos a limitaciones motrices y cognitivas, que ha mostrado mejoras con 2 entrenamientos semanales de 60 minutos en diferentes contextos (Blasco-Lafarga, Monteagudo, Roldán, Cordellat, y Pesce, 2020; Martínez-Navarro, 2014; Monteagudo, Cordellat, Roldán, Gomez-Cabrera, y Blasco-Lafarga, 2019; Roldán et al., 2019). Sus programas, impartidos siempre por técnicos especializados, se vienen realizando en los adultos sanos según el siguiente esquema: 8 meses de entrenamiento, 3 meses de desentrenamiento y 15 días destinados a las evaluaciones, tanto pre como post (1 mes en total). En este estudio ello dio lugar a 2 periodos de entrenamiento (E1 y E2) y 2 de desentrenamiento (D1 y D2), con 5 momentos de muestreo ( Pre $_{1}$, Post $_{1}$, Pre $_{2}$, Post $_{2}$, Pre $_{3}$ ) (Figura 1). Se mantuvieron las dos sesiones semanales, y el programa fue impartido por un equipo estable de 4 especialistas durante los dos años.

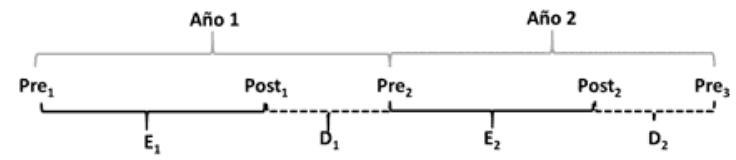

Figura 1. Cronograma del programa EFAM-UV@ a lo largo de los dos años.

Leyenda. Las líneas continuas muestran el periodo de entrenamiento, y las discontinuas e periodo de desentrenamiento. Las uniones de los periodos $\left(\mathrm{Pre}_{1}, \mathrm{Post}_{1}, \mathrm{Pre}_{2}, \mathrm{Post}_{2}, \mathrm{Pre}_{3}\right.$ ) simbolizan los 5 momentos de muestreo.

Como se resume en la figura 2 (esquema básico de la taxonomía EFAM-UV(C), esta metodología persigue mejoras integrales y en 3 ámbitos (neuromuscular, cardiovascular y cognitivo), mejorando la forma física y la alfabetización motriz sobre la reeducación de la marcha y el control postural (Dominios Básicos). Paralelamente establece como dominios complementarios la preservación o mejora de las habilidades manipulativas y cognitivas para consolidar un primer nivel psicomotriz o de Coordinación Primaria que permita seguir mejorando la funcionalidad de sus usuarios. En su segundo nivel (Coordinación precisa con disposición variada) EFAMUV(C busca optimizar la funcionalidad y la plasticidad motriz con tareas complejas como el trabajo de ritmo y un cajón de sastre llamado «destreza motriz», mezcla de juego y habilidad predeportiva. Las tareas están diseñadas para que la dificultad vaya aumentando progresivamente, ajustando al máximo el diseño de las sesiones y los ejercicios a modo de programa periodizado.

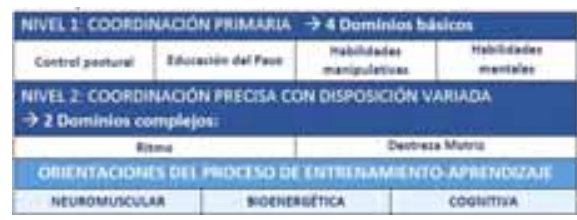

Figura 2. Taxonomía de la metodología EFAM-UV@ (Blasco-Lafarga et al., 2016), programa multicomponente de orientación cognitiva y neuromuscular específico para AM.

La composición corporal y la estatura fueron evaluadas a través de la báscula BC-545N (Tanita; Tokio, Japón) y el tallímetro SECA 222, respectivamente. La agilidad se evaluó mediante el TUG, siguiendo las directrices de Rikli y Jones (1999), contabilizando el tiempo en segundos. Por otro lado, teniendo en cuenta los valores de referencia propuestos por Rikli y Jones (2013) para establecer niveles de independencia

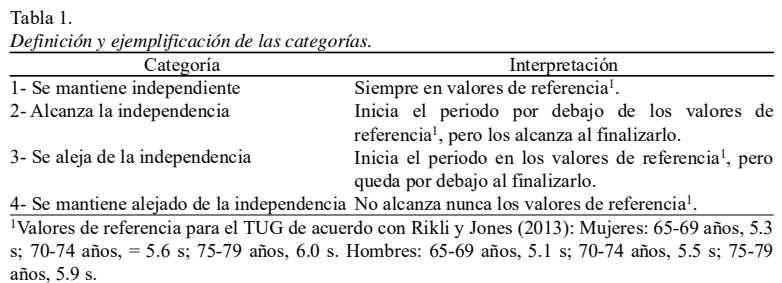


en los adultos mayores y considerando los resultados del TUG en función del género y la edad, se crearon 4 categorías (Tabla 1).

El análisis estadístico se llevó a cabo mediante el programa informático R versión 3.4.2 (2017-09-28). Tras comprobar la normalidad de los datos, se realizó un ANOVA de medidas repetidas y análisis post-hoc (Bonferroni) para comprobar los cambios tras el entrenamiento (E1, E2) y el desentrenamiento (D1, D2), así como, los efectos acumulados del entrenamiento al completo (EnC; cambios de Pre1 a Post2) y la intervención total (Int, Pre1 a Pre3). También se utilizó la asistencia como variable control, realizando así un ANCOVA. Con el fin de homogenizar y analizar los cambios, se calculó el tamaño del efecto, siguiendo las directrices de Cohen (1992) donde se consideró un tamaño del efecto trivial ( $d$ entre $0 \mathrm{y}$ 1.9); pequeño ( $d$ entre .2 y .49); moderado (d entre .5 y .79); grande ( $d$ entre .8 y 1.19); muy grande ( $>1.2$ ). A continuación, se utilizó la prueba del test exacto de Fisher para observar los cambios en la distribución categorial de la muestra a lo largo de los diferentes periodos, atendiendo a las comparaciones con los estándares de independencia funcional. En todos los casos se estableció un valor de alfa de $\mathrm{p}<.05$.

\section{Resultados}

A modo de caracterización general del programa (Tabla 2) encontramos diferencias significativas para la disminución de la masa grasa, con un tamaño del efecto pequeño. El resto de variables tuvieron una magnitud de cambio trivial y no significativa.

\begin{tabular}{|c|c|c|c|c|}
\hline & $\mathrm{Pre}_{1}$ & Post $_{2}$ & $P$ & $d$ \\
\hline Peso $(\mathrm{kg})$ & $66.02(10.03)$ & $65.09(9.15)$ & 0.20 & -0.10 \\
\hline Altura (m) & $1.56(0.06)$ & $1.55(0.06)$ & 0.29 & -0.17 \\
\hline Masa muscular (kg) & $40.96(7.13)$ & $41.35(7.39)$ & 0.55 & 0.05 \\
\hline Masa grasa (\%) & $35.14(5.87)$ & $32.92(5.68)$ & 0.02 & -0.38 \\
\hline Grasa visceral & $11.26(3.26)$ & $10,74(2,81)$ & 0,26 & -0.17 \\
\hline
\end{tabular}

La agilidad (Figura 3) mostró una mejora a lo largo de los dos años ya que el tiempo de ejecución en el test fue disminuyendo. Este cambio fue significativo y grande tras el primer año de entrenamiento, disminuyendo en magnitud en el segundo año ( $d=1.05$ vs .41). Durante el desentrenamiento, la tendencia fue opuesta ya que en el primer periodo el empeoramiento fue menor que en el segundo, aunque con una magnitud pequeña y no significativa $(\mathrm{d}=-.11 \mathrm{vs}-.22)$. Los cambios más grandes y significativos se produjeron entre la comparación del inicio del programa hasta el final del segundo entrenamiento $\left(d_{\mathrm{EnC}}=1.23\right)$ o el inicio del tercer año $\left(d_{\text {Int }}=1.09\right)$. La interacción entre la asistencia y la agilidad no fue significativa.

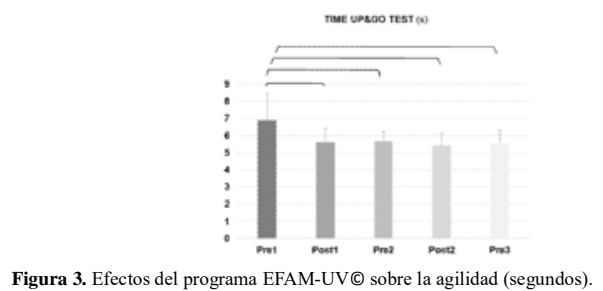

Atendiendo ahora a la distribución de personas en cada categoría, la figura 4 muestra cómo evolucionó la muestra según los cuatro periodos resultantes. Tras el primer

entrenamiento, el $61 \%$ de los participantes se situaron en niveles de independencia funcional (categorías 1 y 2), aumentando al $69 \%$ tras el segundo año. Contrariamente a la situación ideal, y a pesar del entrenamiento un 5\% se alejó de la independencia en E2 (categoría 3). En cuanto al desentrenamiento (figura 4), EFAM-UVC tuvo un bajo efecto residual sobre la agilidad en su primer periodo. En este periodo aumentó el porcentaje de los dependientes (categoría 3 y $4=$ $60 \%$ ), y esto provocó que la agilidad no situara a la mitad de los participantes en la zona de independencia funcional. Sin embargo, esta tendencia se invierte en el segundo año, ya que sólo se quedaron fuera de la independencia un $43 \%$ de participantes. Esta capacidad consiguió situar a personas en la categoría 2 en los dos momentos de desentrenamiento, y reducir el número de personas que se alejaron de la independencia tras el D2.

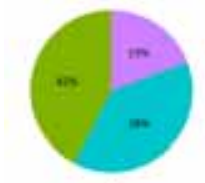

a

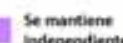

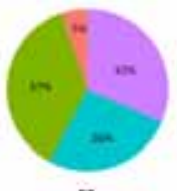

e

Independ

Figura 4. Categorización de la agilidad.

Leyenda. Efectos del primer y segundo periodo de Entrenamiento (E1 y E2) y Desentrenamiento (D1 y D2) según los valores normativos de Rikli y Jones (2013) para edad y género.

Por último, analizando la distribución se encontró una tendencia a la significatividad cuando se comparó la distribución del primer entrenamiento (E1) frente el primer desentrenamiento $(D 1)(p=.06)$. Y diferencias significativas cuando se comparó E1 frente a D2 $(\mathrm{p}=.01)$.

\section{Discusión}

El principal hallazgo fue que la agilidad mejoró de forma importante a lo largo del primer periodo de entrenamiento, mostrando unas pérdidas muy pequeñas durante el desentrenamiento. Ello provocó que al final de los dos años se obtuviera un beneficio aún mayor en relación al punto de partida, y pudieran alcanzar la zona de independencia funcional más de la mitad de los participantes, a pesar de que eran 2 años mayores.

La comparación por periodos mostró una mejora con un tamaño del efecto grande en su primer entrenamiento y pequeño en su segundo entrenamiento. En la literatura encontramos que Neto, Santos, Silva, de Santana, y Da SilvaGrigoletto (2018) con un programa de entrenamiento funcional (3 días/semana; 64 años de media), consiguieron una magnitud de cambio en su primer periodo de entrenamiento de 1.40. También Leitão et al. (2015), esta vez con un entrenamiento multicomponente ( 2 días/semana; 66,7 años de media), consiguieron unas ganancias mayores (1.51). Es posible que la edad sea una de las causas por las cuales podemos encontrar dicha diferencia en el tamaño del efecto. O también, la asistencia al programa, ya que en los dos estudios comentados fue del $80 \%$ y en nuestro estudio fue del $71.6 \%$, ya que se ha aceptado que aquellos estudios con una duración superior a 6 meses, la asistencia se puede reducir como mínimo a un 69.1\% (Farrance, Tsofliu, y Clark, 2016).

Durante el segundo periodo de entrenamiento, Leitão et 
al. (2015) obtuvieron el mismo tamaño del efecto que nosotros (0.41). La atenuación durante el segundo periodo de entrenamiento puede ser debido a las adaptaciones fisiológicas producidas por el primer periodo. Esta tendencia se ve corroborada gracias a estudios como el de Virág, Harkányi, Karóczi, Vass, y Kovács (2018) donde compararon la evolución en los resultados de 3 grupos: un grupo no entrenado, un grupo que había entrenado máximo 6 meses, y otro que había entrenado durante más de 6 meses. Y, efectivamente, en este último grupo, las mejoras fueron las más pequeñas respecto a los otros grupos.

Un punto en común que tienen los estudios descritos (entrenamiento multicomponente y funcional) junto con el nuestro es la similitud con las tareas llevadas a cabo. Es decir, para mejorar la agilidad, Donath et al. (2016) establecieron que los ejercicios debían comprender: tareas complejas, cambios de dirección y toma de decisiones, de forma similar a la metodología EFAM-UVC.

Centrándonos ahora en el análisis sobre el desentrenamiento, las pérdidas fueron triviales y pequeñas en su primer y segundo periodo de desentrenamiento, respectivamente. Leitão et al. (2015) mostraron pérdidas con un tamaño del efecto de -0.6 frente al -0.11 en nuestro estudio. Este resultado es muy importante ya que conseguir retener las adaptaciones provocadas es uno de los objetivos fundamentales en el entrenamiento de esta población. Respecto al segundo desentrenamiento, el programa EFAMUV(C) consigue mantener valores similares respecto al programa anteriormente comentado, y es que ellos obtuvieron pérdidas de -0.29 y nuestros participantes de -0.22 . Es decir, las pérdidas del primer periodo de desentrenamiento fueron menores a las del segundo, en contra de la hipótesis planteada al inicio de la investigación.

Cabe destacar que dos años después de haber empezado el programa de entrenamiento EFAM-UVC el tamaño del efecto fue muy grande, por tanto, la capacidad de mejora sobre la agilidad es un hecho a destacar por su transferencia en la vida de los participantes.

El análisis por categorías mostró que más de la mitad de los participantes consiguieron situarse en valores de mantener o alcanzar la independencia, ratificando así la mejora mostrada por las comparaciones por pares. Cabe destacar que, a pesar de que el segundo año no se alcanzaron las mismas mejoras, sí que se pudo aumentar el número de personas con independencia funcional, además de disminuir frente al primer periodo aquellas que no lo consiguieron, de forma contraria a la hipótesis establecida. Estos resultados son interesantes para promover con más énfasis el ejercicio físico entre adultos mayores, por su repercusión sobre la posible disminución de gastos sanitarios. Se utilizaron los valores de referencia americanos por ser los más extendidos, pero si los comparamos frente a los españoles, observamos que estos últimos son menos exigentes. Es decir, para situarse en la zona de independencia funcional según Rikli y Jones (2013), los valores de la población española se encuentran todos en el percentil 95, aun encontrando valores más exigentes para la muestra americana, alrededor de 0.2-0.3 segundos. Es por ello que, a pesar de que sea una capacidad que se mejore mucho tras el entrenamiento, no es suficiente como para alcanzar los valores de la población americana. Es por ello, que los estándares de la agilidad en los valores de referencia son exigentes y merecen ser revisados en la población española, pues pueden no estar reflejando bien las mejoras obtenidas tras los programas de entrenamiento. Por tanto, deben ser tomados con cautela.

Generalmente se utilizan pruebas t para el análisis de los programas, pero una información muy valiosa que enmascara esta prueba es si los adultos mayores consiguen alcanzar la independencia o no. Es por ello, que el análisis categorial permite dar respuesta a esta pregunta que se complementa con la primera, ya que para los técnicos tan importante es saber que sus participantes mejoran como conocer quiénes son los participantes que se sitúan en valores por debajo de lo esperado en relación a su género y edad, para reforzar las tareas y ofrecerles el estímulo óptimo.

\section{Conclusiones}

Como conclusión, se remarca la necesidad de utilizar una comparación u otra en función de los objetivos. Si se quiere observar la cantidad de mejora, habrá que utilizar el primer análisis, aunque si se pretende esclarecer la mejora según la independencia funcional, será necesario realizar el segundo.

Por otro lado, la principal limitación fue la falta de grupo control. Y, el posible efecto aprendizaje a lo largo de los diferentes momentos de muestreo. Como futuras líneas de investigación, se deberían consolidar los percentiles en la población española y, sobre todo, continuar analizando los entrenamientos en función de las categorías para detectar a aquellos participantes que necesitan más de dos días de entrenamientos o más intensidad durante el entrenamiento.

\section{Referencias}

Bauman, A., Merom, D., Bull, F., Buchner, D., \& Fiatarone Singh, M. (2016). Updating the Evidence for Physical Activity: Summative Reviews of the Epidemiological Evidence, Prevalence, and Interventions to Promote «Active Aging». Gerontologist, 56(S2), S268-S280. doi: 10.1093/geront/gnw031

Blasco-Lafarga, C., Martinez-Navarro, I., Cordellat,A., Roldán, A., Monteagudo, P., Sanchis-Soler, G., \& Sanchis-Sanchis, R. (2016). Método de Entrenamiento Funcional Cognitivo Neuromotor. Propiedad Intelectual $n^{\circ} 156069$, España (2016).

Blasco-Lafarga, C., Monteagudo, P., Roldán, A., Cordellat, A., \& Pesce, C. (2020). Strategies to change body composition in older adults: Do type of exercise and dose distribution matter? Journal of Sports Medicine and Physical Fitness (In press).

Bouaziz, W., Lang, P., Schmitt, E., Kaltenbach, K., Geny, B., \& Vogel, T. (2016). Health benefits of multicomponent training programmes in seniors: a systematic review. International Journal Clinical Practice, 70(7): 520-536. doi: $10.1111 /$ ijcp. 12822

Cohen, J. (1992). A power primer. Psychological Bulletin, 112(1), 155-159.

Donath, L., van Dieën, J., \& Faude, O. (2016). Exercise-Based Fall Prevention in the Elderly: What About Agility? Sports Medicine, 46(2), 143-149. doi: 10.1007/s40279-015-0389- 
Eggenberger, P., Theill, N., Holenstein, S., Schumacher, V., \& de Bruin, E. D. (2015). Multicomponent physical exercise with simultaneous cognitive training to enhance dualtask walking of older adults: a secondary analysis of a 6month randomized controlled trial with 1-year follow-up. Clinical Interventions Aging, 10, 1711-1732. doi: 10.2147/ CIA.S91997

Farrance, C., Tsofliu, F., \& Clark, C. (2016). Adherence to community based group exercise interventions for older people: A mixed-methods systematic review. Preventive Medicine, 87, 155-166. doi: 10.1016/j.ypmed.2016.02.037

Fritz, S., \& Lusardi, M. (2009). Walking Speed: the Sixth Vital Sign. Journal of Geriatric Physical Therapy, 32(2), 2-5.

Gerard, K., Salicetti, A., Moncada, J., \& Solano, L. (2018). Mejora del equilibrio, atención y concentración después de un programa de entrenamiento exergame en la persona adulta mayor. Retos. Nuevas tendencias en Educación Física, Deporte y Recreación, 33, 102-105.

Lee, J., Chun, H., Kim, Y.-S., Jung, H.-W., Jang, I.-Y., Cha, H., ... Yoon, J. (2020). Association between Timed Up and Go Test and Subsequent Functional Dependency. Journal of Korean Medical Science, 35(3), 1-11.

Leitão, L., Brito, J., Leitão, A., Pereira, A., Conceição, A., Silva, A., \& Louro, H. (2015). Functional capacity retention in older women after multicomponent exercise cessation: 3year longitudinal study. Motricidade, 11(3), 81-91.

Martínez-Heredia, N., Santaella, E., \& Rodríguez-García,A. (2021). Beneficios de la actividad física para la promoción de un envejecimiento activo en personas mayores. Revisión bibliográfica. Retos. Nuevas tendencias en Educación Física, Deporte y Recreación, 39, xx-xx.

Martínez-Navarro, I. (2014). Efectos de un programa de entrenamiento funcional sobre la variablidad de la frecuencia cardíaca, la función ejecutiva y la capacidad condicional en los adultos mayores. (Doctoral dissertation). Universitat de València., Valencia.

Monteagudo, P., Cordellat, A., Roldán, A., Gomez-Cabrera, M. C., \& Blasco-Lafarga, C. (2019). Effects of multicomponent exercise on metabolic health parameters in elderly. MOJ Sports Med, 3(3), 70-74.

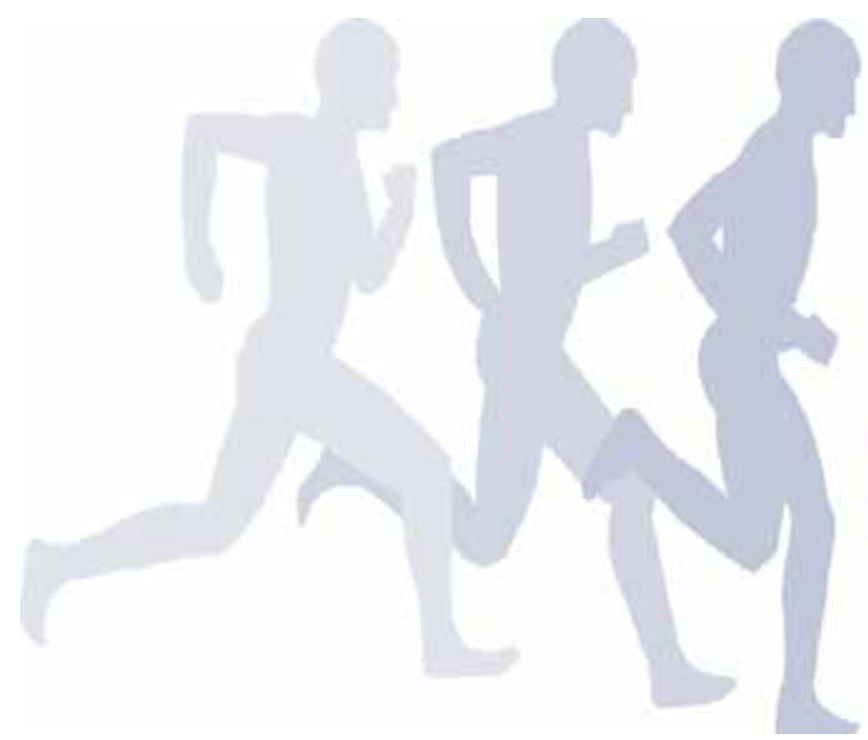

Neto, A., Santos, M., Silva, R., de Santana, J., \& Da SilvaGrigoletto, M. (2018). Effects of different neuromuscular training protocols on the functional capacity of elderly women. Revista Brasileira Medicina do Esporte, 24(2), 140-144.

Pedersen, B. K., \& Saltin, B. (2015). Exercise as medicineevidence for prescribing exercise as therapy in 26 different chronic diseases. Scandinavian Journal of Medicine and Science in Sports, 25, 1-72. doi: 10.1111/sms. 12581

Rikli, R. E., \& Jones, C. J. (1999). Development and validation of a functional fitness test for community-residing older adults. Journal of Aging and Physical Activity, 7(2), 129161. doi: 10.1123/japa.7.2.129

Rikli, R. E., \& Jones, C. J. (2013). Development and Validation of Criterion-Referenced Clinically Relevant Fitness Standards for Maintaining Physical Independence in Later Years. The Gerontologist, 53(2), 255-267. doi: 10.1093/geront/gns071

Roldán, A., Cordellat, A., Monteagudo, P., García-Lucerga, C., Blasco-Lafarga, N. M., Gomez-Cabrera, M. C., \& Blasco-Lafarga, C. (2019). Beneficial Effects of Inspiratory Muscle Training Combined With Multicomponent Training in Elderly Active Women. Research quarterly for exercise and sport, 90(4), 547-554.

Salinas-Martínez, F., Cocca, A., Mohamed, K., \& Viciana, J. (2010). Actividad Física y sedentarismo: Repercusiones sobre la salud y calidad de vida de las personas mayores. Retos. Nuevas tendencias en Educación Física, Deporte y Recreación, 17, 126-129.

Sampedro, J., Meléndez, A., \& Ruiz, P. (2010). Análisis comparativo de la relación entre el número de caídas anual y baterías de pruebas de equilibrio y agilidad en personas mayores. Retos. Nuevas tendencias en Educación Física, Deporte y Recreación, 17, 115-117.

Virág, A., Harkányi, I., Karóczi, C., Vass, Z., \& Kovács, É. (2018). Study of effects of multimodal exercise programme on physical fitness and health perception in communityliving Hungarian older adults. The Journal of Sports Medicine and Physical Fitness.

Wiles, J. L., Leibing, A., Guberman, N., Reeve, J., \& Allen, R. E. (2012). The meaning of «aging in place» to older people. The Gerontologist, 52(3), 357-366.

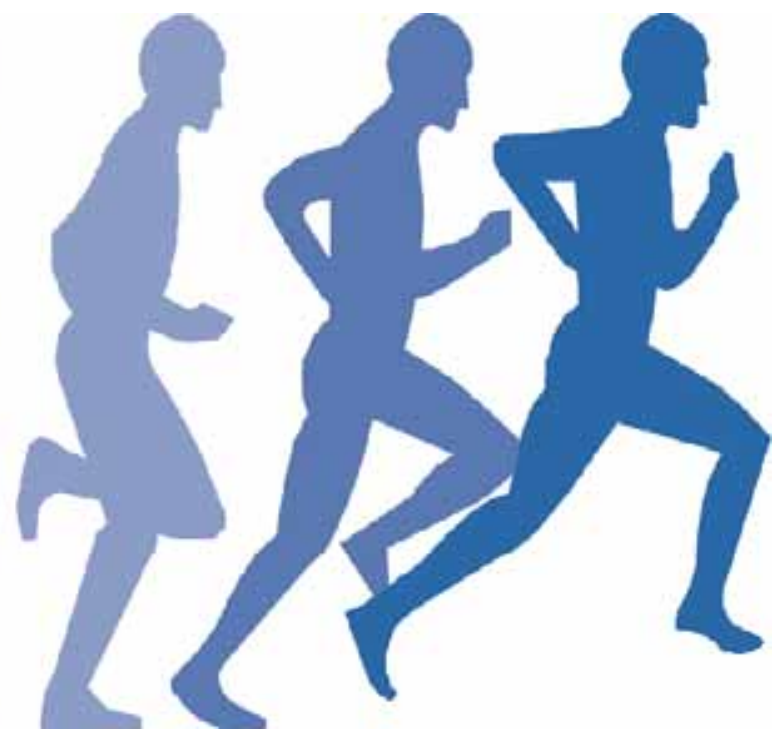

